\title{
EFEITO DO TRATAMENTO DE IMPLANTAÇÃO IÔNICA POR IMERSÃO EM PLASMA NA RESISTÊNCIA AO DESGASTE DA LIGA Ti-6Al-4V*
}

\author{
Susana Zepka' \\ Maria Margareth da Silva ' \\ Danieli Aparecida Pereira Reis ${ }^{2}$ \\ Mário Ueda ${ }^{3}$ \\ Adriano Gonçalves dos Reis '
}

\section{Resumo}

O objetivo deste trabalho foi a avaliação da resistência ao desgaste da liga Ti-6Al-4V após processo de Implantação lônica por Imersão em Plasma (IIIP) em diferentes tempos de imersão. A finalidade deste processo foi a modificação das propriedades superficiais da liga Ti-6Al-4V, com intuito de se obter melhorias nas suas propriedades tribológicas. Nesse processo, átomos podem ser injetados na superfície dos materiais, modificando suas propriedades mecânicas na região próxima à superfície independentemente de variáveis termodinâmicas, tais como solubilidade e difusividade. Foram analisadas amostras submetidas a tempos de imersão de 120 e 180 minutos, a temperaturas de $250^{\circ} \mathrm{C}$ em 3 amostras para cada condição estudada. A análise de desgaste foi realizada pelo processo pin-on-disk, onde foram comparados os volumes perdidos e os coeficentes de desgaste das amostras. A amostra imersa por 180 minutos teve um coeficiente de desgaste com relação à amostra sem tratamento de 35, I 2\% menor, e em relação a amostra de 120 minutos foi de I I,09\% menor. Pode-se observar que a amostra com maior tempo de imersão apresentou menor coeficiente de desgaste.

Palavras-chave: Fluência; Ti-6Al-4V; IIIP; Desgaste.

\section{EFECT OF PLASMA IMMERSION ION IMPLANTATION TREATEMENT IN THE WEAR RESISTANCE OF Ti-6AI-4V ALLOY}

\begin{abstract}
The objective of this work was the evaluation of wear resistance of Ti-6Al-4V alloy after plasma immersion ion implantation (PIII) in different immersion times. The goal of this process was the modification of surface properties of the alloy to obtain better tribology properties. In this process, atoms can be injected on the material's surface changing the mechanical properties in the region near the surface independently of thermodynamics variables, as solubility and difusivity. The samples were submitted to 120 e 180 minutes of implantation at $250^{\circ} \mathrm{C}$ in the three samples for each condition. The wear analyses were made by pin-on-disk process, where the lost volumes and wear coefficients were compared in the samples. It was observed the decreasing of attrite coefficient and the lost volume of the material during wear test. The implanted sample by 180 minutes has showed the wear coefficient $35.12 \%$ lower in comparison of the sample without treatment, and $11.09 \%$ lower in comparison of implanted sample by 120 minutes. It can be observed that the sample implanted by 180 minutes showed lower wear coefficient.
\end{abstract}

Keywords: Creep; Ti-6Al-4V; PIII; Wear.

*Dedicado à Memória do Prof. Carlos de Moura Neto.

\footnotetext{
IInstituto Tecnológico de Aeronáutica - ITA, São José dos Campos, SP, Brasil. E-mail: meg@ita.br

${ }^{2}$ Universidade Federal de São Paulo - UNIFESP, São José dos Campos, SP, Brasil.

${ }^{3}$ Instituto Nacional de Pesquisas Espaciais - INPE, São José dos Campos, SP, Brasil.
} 


\section{INTRODUÇÃO}

Titânio e suas ligas têm um lugar de destaque no desenvolvimento de materiais, com tratamento superficial, para trabalhos em ambientes agressivos tanto da indústria aeronáutica, química, petrolífera ou para uso como material bio compatível. Uma das ligas de titânio mais utilizada é a Ti-6Al-4V devida a suas propriedades mecânicas, dureza, resistência a corrosão e estabilidade química $[1,2]$. Porém, existem fatores que limitam a aplicação do Ti e suas ligas como material estrutural, principalmente em temperaturas elevadas, um deles é sua avidez por oxigênio [3]. $O$ uso destes materiais em temperaturas acima de $600^{\circ} \mathrm{C}$, causam problemas com a oxidação superficial.

Portanto, faz-se necessário o estudo de tratamentos superficiais que diminuam as limitações impostas. A tecnologia emergente da Implantação lônica por Imersão em Plasma vem trazer uma técnica razoavelmente simples, econômica e podendo ser usada em estruturas de perfil complexo em 3D, esta técnica tem tido uma atenção especial dos pesquisadores, pois a superfície não depende de aderência de camadas e nem formação de filmes [4-9].

O objetivo deste estudo foi qualificar a melhoria do tratamento superficial da liga quanto ao desgaste em tempos de imersão de 120 e 180 minutos.

\section{MATERIAIS E MÉTODOS}

\section{I Material e Corpos de prova}

Para a realização deste trabalho, foi utilizada a liga Ti-6Al-4V adquirida da empresa Multialloy Metais e Ligas Especiais Ltda na forma de barras cilíndricas de 12,7 mm de diâmetro e I,0 m de comprimento e na condição forjada e recozida a $190^{\circ} \mathrm{C}$ durante 6 horas e resfriada ao ar, apresentando estrutura equiaxial. Foi utilizado a liga em forma de pastilhas, de $10 \mathrm{~mm}$ de diâmetro e $3 \mathrm{~mm}$ de espessura, polidas com acabamento espelhado, limpas por ultrassom em acetona por 5 minutos.

\subsection{Processo de Implantação lônica por Imersão em Plasma (IIIP)}

A técnica de Implantação lônica por Imersão em Plasma consistiu na implantação de íons, no caso de nitrogênio, na superfície de um corpo de prova. A Figura I apresenta o que ocorre na superfície do material durante o tratamento de implantação iônica, observando-se o caminho percorrido pelo íon implantado.

O processo de IIP inicia-se com a montagem do corpo de prova de Ti-6Al-4V no dispositivo de suporte de amostras, introduzido e fixado no reator. Este é selado para a formação do vácuo, visando o tratamento superficial, através de uma bomba mecânica e posteriormente uma bomba difusora até atingir a pressão de até $5,3 \times 10^{-3} \mathrm{mbar}$, quando o sistema está pronto para iniciar o processo de implantação. De acordo com o esquema da Figura 2, foi injetado nitrogênio (para a implantação de íons de nitrogênio na amostra). Para obtenção do plasma, ligou-se o pulsador RUP-4. Os tratamentos de implantação de nitrogênio foram feitos a $250^{\circ} \mathrm{C}$, pulso de alta voltagem de $8,5 \mathrm{kV}$, amplitude média de duração de $40 \mu \mathrm{s}$, frequência de $400 \mathrm{~Hz}$, pressão de trabalho de 0,2I Pa por 120 e 180 minutos [9-II].

\subsection{Análise de Desgaste}

A análise de desgaste foi feita através do método pino sobre disco (pin-on-disk), cujo esquema experimental está representado na Figura 30 equipamento utilizado foi o “CSM-Instruments Pin-on-disk Tribometer", SN I8-3 I3, pertencente ao LAP (Laboratório Associado de Plasma), do INPE.

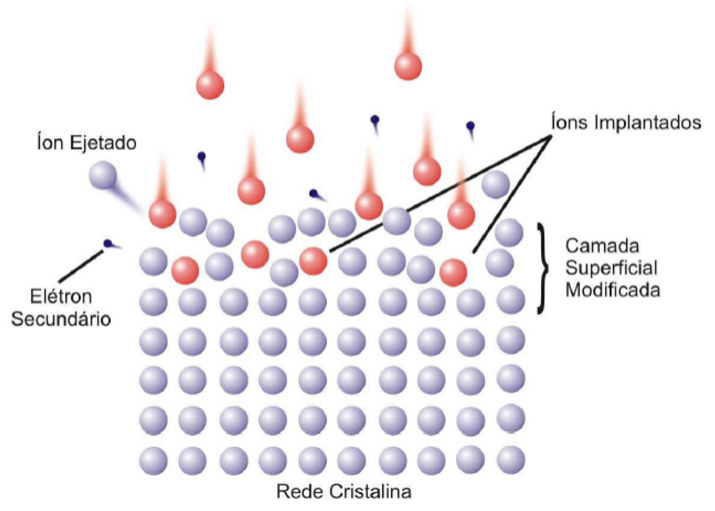

Figura I. Representação do processo de implantação iônica [9].

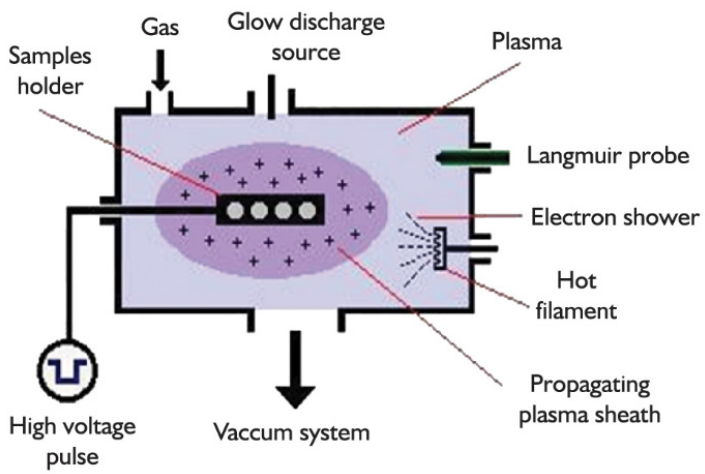

Figura 2. Esquema do equipamento de IIIP.

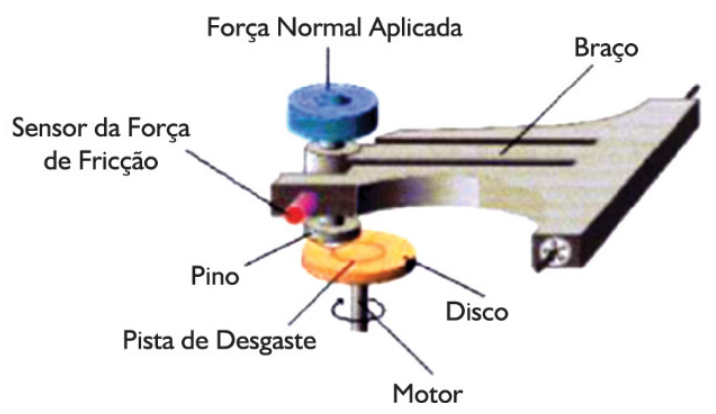

Figura 3. Esquema do equipamento de medição de desgaste. 
O desgaste superficial das amostras foi medido segundo a Norma ASTM G99-95a [12], assim como o coeficiente de atrito. A amostra como recebida, sem tratamento IIIP, foi usada como referência para a análise de caracterização.

A largura da trilha de desgaste foi medida via microscópio óptico, obtendo-se a média por trecho de trilha. A Figura 4 mostra a imagem da trilha observada no microscópio óptico para uma amostra com tempo de implantação de 120 min.

A limpeza das amostras e do pino foi feita com acetona, a análise foi realizada na temperatura ambiente $\left(18^{\circ} \mathrm{C}\right.$ a $\left.22^{\circ} \mathrm{C}\right)$ e umidade relativa entre $50 \%$ e $70 \%$. O pino foi esférico de alumina policristalina, com $99,85 \%$ de pureza, com dureza de $19.000 \mathrm{~N} / \mathrm{mm}^{2}$ e diâmetro de $3 \mathrm{~mm}$. A carga normal utilizada foi de $\mathrm{I}, 0 \mathrm{~N}$, raio da pista de desgaste de $3 \mathrm{~mm}$, distância percorrida 47, I $3 \mathrm{~m}$ (5.000 voltas), taxa de aquisição de dados de $5 \mathrm{~Hz}$ e velocidade de $5 \mathrm{~cm} / \mathrm{s}$

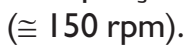

O desgaste é determinado através da medida da largura da trilha de desgaste, que deve ser convertida para volume de desgaste em $\mathrm{mm}^{3}$, também chamada de volume perdido (V), e calculado de acordo com Equação l:

$$
V=2 \pi R\left[r^{2} \operatorname{sen}^{-1}(d / 2 r)-(d / 4)\left(4 r^{2}-d^{2}\right)^{1 / 2}\right]
$$

Onde: $\mathrm{R}=$ raio da trilha de desgaste, $\mathrm{d}=$ largura da trilha de desgaste e $r=$ raio do pino.

E o coeficiente de desgaste $\mathrm{K}$ é calculado através da Equação 2:

$$
\mathrm{K}=\mathrm{V} /(\mathrm{N} . \mathrm{L})
$$

Onde: $\mathrm{V}=$ volume perdido, $\mathrm{N}=$ distância percorrida em metros e $\mathrm{L}=$ carga aplicada em Newtons.

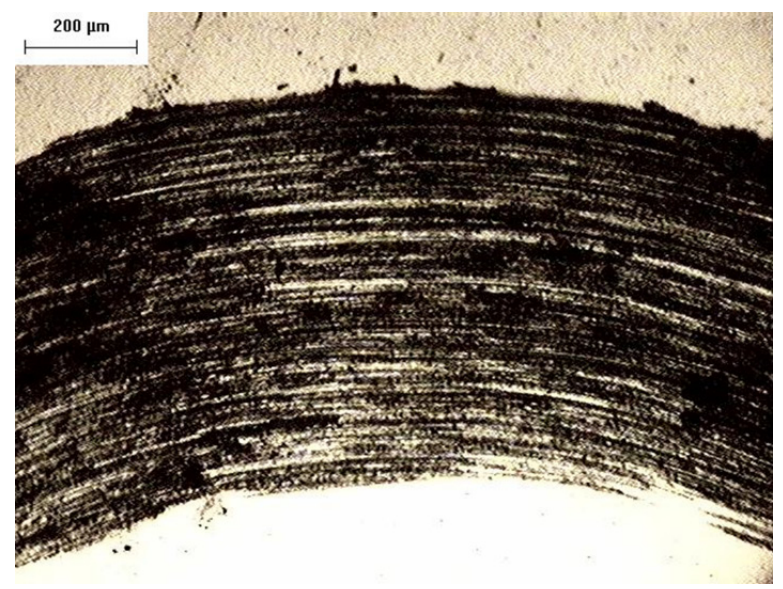

Figura 4. Imagem da trilha no microscópio óptico de uma amostra implantada por 120 min.

\section{RESULTADOS E DISCUSSÕES}

Tem-se os valores de $\mathrm{V}$ e K para cada tempo de imersão mostrados na Tabela I. Pode-se observar que a amostra com maior tempo de imersão apresentou menor coeficiente de desgaste.

O resultado do teste de desgaste é mostrado na Figura 5, onde pode-se observar que o coeficiente de atrito da amostra tratada é função do número de ciclos, e a partir de determinado número de ciclos a média do coeficiente de atrito da amostra com 120 minutos de implantação se iguala a da amostra sem tratamento que é de 0,47.

A diferença dos valores de coeficiente de atrito entre as amostras nos primeiros 1.000 ciclos se dá pela modificação das superfícies pelo tratamento aplicado, onde uma nano camada implantada com íons de nitrogênio endurece a
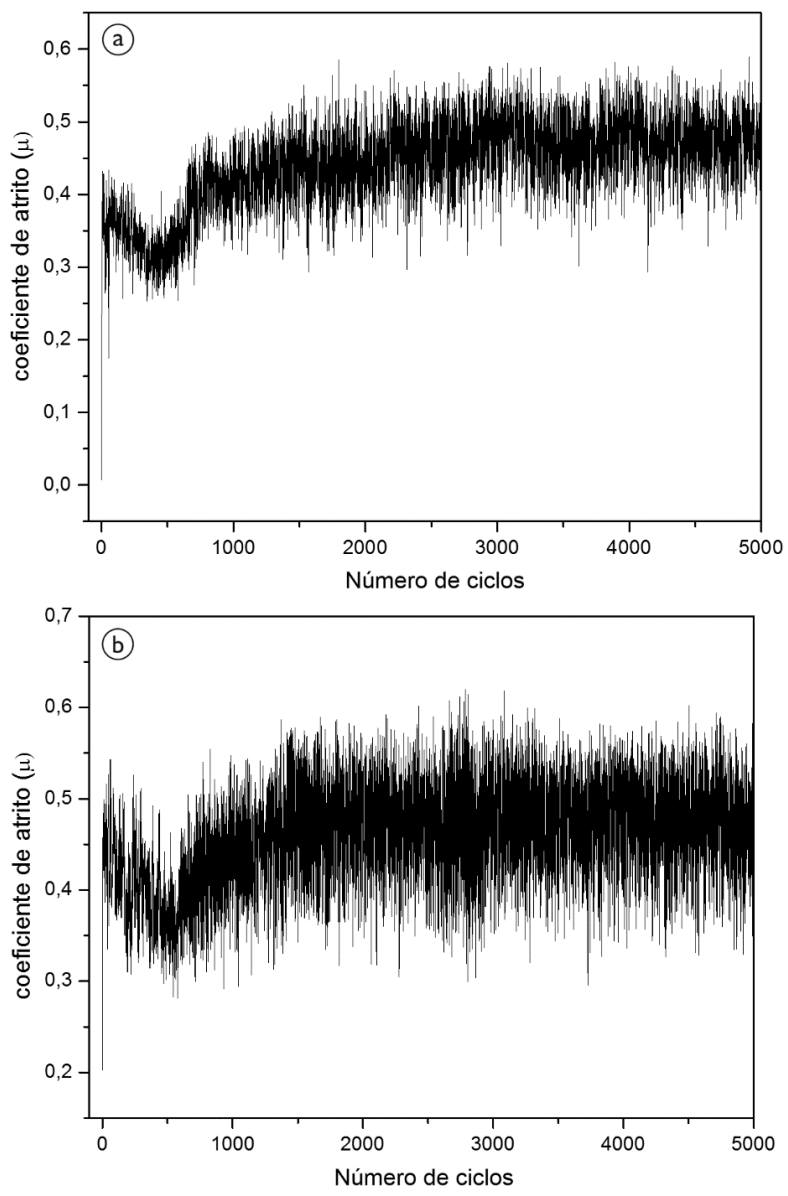

Figura 5. Relação de coeficiente de atrito e número de ciclos em uma amostra sem tratamento (a) e tratada com imersão por plasma por 120 minutos (b).

\begin{tabular}{|c|c|c|c|}
\hline Tempo de Imersão (min) & Largura da Trilha - d (mm) & Volume Perdido - V $\left(\mathrm{mm}^{3}\right)$ & $\begin{array}{c}\text { Coeficiente de Desgaste - K } \\
10^{-3}\left(\mathrm{~mm}^{3} / \mathrm{N} . \mathrm{m}\right)\end{array}$ \\
\hline 0 & 0,7373 & 0,4279 & 9,0791 \\
\hline 120 & 0,6636 & 0,3101 & 6,5797 \\
\hline 180 & 0,6434 & 0,2776 & 5,8901 \\
\hline
\end{tabular}

Tabela I. Valores de $\mathrm{V}$ e K para cada tempo de imersão. 
superfície diminuindo seu coeficiente de atrito, porém seu núcleo permanece o mesmo da amostra sem tratamento.

Comparando a amostra sem tratamento com a imersa em plasma por 120 minutos, pode-se notar que o coeficiente de desgaste foi $27,53 \%$ menor na amostra tratada. A amostra imersa por 180 minutos teve um coeficiente de desgaste com relação à amostra sem tratamento de 35, I 2\% menor, e em relação a amostra de 120 minutos foi de II,09\% menor. Este resultado está coerente, pois quanto menor o coeficiente de desgaste, mais duro é o material e, portanto menos material é perdido $[6,13]$. Comparando os resultados a outros obtidos em diversos trabalhos, pode-se dizer que foram compatíveis com o esperado [|4-|8].

\section{CONCLUSÕES}

O tratamento superficial com a implantação iônica por imersão em plasma é um método bastante atraente para modificações superficiais provendo endurecimento progressivo da superfície conforme aumenta-se o tempo de imersão, isto se deve ao aumento de tensão na superfície na implantação dos íons implantados e também pela formação de TiN [19]. Devido a estes fatores observou-se a diminuição do coeficiente de atrito e do volume perdido de material durante os ensaios de desgaste. A amostra imersa por 180 minutos teve um coeficiente de desgaste com relação à amostra sem tratamento de 35, I 2\% menor, e em relação a amostra de 120 minutos foi de I I,09\% menor. Pode-se observar que a amostra com maior tempo de imersão apresentou menor coeficiente de desgaste.

\section{Agradecimentos}

CAPES, CNPq e FAPESP pelo suporte financeiro.

\section{REFERÊNCIAS}

I Sakai T, Ohashi M, Chiba K, Jonas JJ. Recovery and recrystallization of polycrystalline nickel after hot working. Acta Metallurgica. 1988;36(7): I 781-1790. http://dx.doi.org/10.1016/0001-6 I60(88)90246-5.

2 Lee WS, Lin CF. High-temperature deformation behavior of Ti-6Al-4V alloy evaluated by strain-rate compression tests. Journal of Materials Processing Technology. 1998;75(I-3): I 27-I36. http://dx.doi.org/I0. I0I6/S0924$0136(97) 00302-6$.

3 Welsch G, Kahveci Al. Oxidation of high temperature: intermetallics. Warrendale: TMS; 1988.

4 Silva MM, Ueda M, Pichon L, Reuther H, Lepienski CM. Surface modification of Ti-6Al-4V alloy by PIII at high temperatures: Effects of plasma potential. Nuclear Instruments \& Methods in Physics Research. Section B, Beam Interactions with Materials and Atoms. 2007;257(I-2):722-726. http://dx.doi.org/I0.10I6/j.nimb.2007.0 I.I35.

5 Ueda M, Silva MM, Lepienski CM, Soares PC Jr, Gonçalves JAN, Reuther H. High Temperature Plasma Immersion Ion Implantation of Ti6Al4V. Surface and Coatings Technology. 2007;20 I (9-I I):4953-4956. http://dx.doi. org/10.1016/j.surfcoat.2006.07.074.

6 Mello CB, Ueda M, Silva MM, Reuther H, Pichon L, Lepienski CM. Tribological effects of plasma immersion ion implantation heating treatments on Ti-6Al-4V alloy. Wear. 2009;267(5-8):867-873. http://dx.doi.org/l0.1016/j. wear.2008.12.103.

7 Reis DAP. Efeito do recobrimento cerâmico e da atmosfera de ensaio na fluência de liga refratária [tese de doutorado]. São José dos Campos: Instituto Nacional de Pesquisas Espaciais; 2004.

8 Silva MM. Modificação de propriedades superficiais da liga Ti-6Al-4V por processos assistidos a plasma, em baixas e altas temperaturas [tese de doutorado]. São José dos Campos: Instituto Tecnológico da Aeronáutica; 2007.

9 Savonov GS. Implantação lônica por Imersão em Plasma em materiais metálicos leves [tese de doutorado]. São José dos Campos: Instituto Nacional de Pesquisas Espaciais; 2010.

I0 Fouquet V, Pichon L, Straboni A, Drouet M. Nitridation of Ti6Al4V by PBIl: study of the nitrogen diffusion and of the nitride growth mechanism. Surface and Coatings Technology. 2002; I86(I-2):34-39. http://dx.doi.org/I0.I0I6/j. surfcoat.2004.04.006.

I I Fouquet V, Pichon L, Drouet M, Straboni A. Plasma assisted nitridation of Ti-6Al-4V. Applied Surface Science. 2004;22 I (I -4):248-258. http://dx.doi.org/10.1016/S0 I69-4332(03)00889-4.

12 American Society for Testing and Materials - AS|TM. ASTM G99-95a: standart test method for wear testing with a pin-on-disk apparatus. West Conshohocken; 2000.

I 3 Zepka S, Reis DAP, Silva MM, Ueda M, Lepienski CM, Neto CM. Study of Creep and Nanoindentation of Ti-6al-4v Alloy after Plasma Immersion lon Implantation (PIII). In: Sociedade Portuguesa de Materiais. Proceedings of Materiais 2013 Congress - International; 20I3, March 25-27; Coimbra, Portugal. Coimbra: SPM; 2013. 
14 Ueda M, Silva MM, Otani C, Reuther H, Yatsuzuka M, Lepienski CM, et al. Improvement of tribological properties of Ti6Al4V by nitrogen plasma immersion ion implantation. Surface and Coatings Technology. 2003; 169-I70:4084I0. http://dx.doi.org/I0.1016/S0257-8972(03)00I37-3.

15 Silva LIG, Ueda M, Silva MM, Coaro EN. Results from Experiments on Hybrid Plasma Immersion lon Implantation/ Nitriding Processing of Materials. IEEE Transactions on Plasma Science. 2004;221:34-39.

16 Silva MM, Ueda M, Otani C, Reuther H, Lepienski CM, Soares PC Jr, et al. Hybrid Processing of Ti-6Al-4V Using Plasma Immersion Ion Implantation Combined with Plasma Nitriding. Materials Research. 2006;9(I):97-I00. http:// dx.doi.org/10.1590/S1516-14392006000100018.

17 Rabinowicz E. Friccion and wear of materials. Massachusetts: John Wiley; 1964.

I8 Silva MM, Pichon L, Otubo J. Wear properties studies of NiTi shape memory alloy after nitrogen plasma based treatments. In: Associação Brasileira de Engenharia e Ciências Mecânicas. Proceedings of 2 Ist International Congress of Mechanical Engineering - COBEM; 201 I October 24-28; Natal, Brazil. São Paulo: ABCM; 201 I.

19 Raveh A. Mechanisms of r. f. plasma nitriding of Ti-6Al-4V alloy. Materials Science and Engineering A. 1993;167(I-2):I55-164. http://dx.doi.org/I0.1016/092I-5093(93)90349-J.

Recebido em: 20 Nov. 2013

Aceito em: 20 Mar. 2015 